Pacific Journal of Mathematics

COMPARISON THEOREMS FOR SECOND-ORDER
OPERATOR-VALUED LINEAR DIFFERENTIAL EQUATIOn 


\title{
COMPARISON THEOREMS FOR SECOND-ORDER OPERATOR-VALUED LINEAR DIFFERENTIAL EQUATIONS
}

\author{
G. J. Butler AND L. H. ERBe
}

Let $B$ be a Banach lattice with order continuous norm, $\mathcal{L}(B)$ the algebra of bounded linear operators. Let $B_{+}$denote the positive cone induced by the lattice structure of $B$, and $\mathcal{L}_{+}(B)$ the corresponding positive cone in $\mathcal{L}(B)$. We consider second-order operator-valued differential equations of the form $Y^{\prime \prime}+Q(x) Y=0$, where $Q:[a,+\infty) \rightarrow$ $\mathcal{L}(B)$ is continuous in the uniform topology and is such that $\int_{x}^{\infty} Q(t) d t$ $\in \mathcal{L}_{+}(B)$ for all $x \geq a$. Comparison theorems of Hille-Wintner type are obtained.

1. Introduction. Consider the second-order linear differential equation

$$
Y^{\prime \prime}+Q(t) Y=0
$$

where $Q:[a,+\infty) \rightarrow \mathcal{L}(B)$ is a continuous operator-valued function and $\mathcal{L}(B)$ represents the Banach algebra of bounded linear operators $T$ : $B \rightarrow B$, where $B$ denotes a Banach space. By a solution $Y$ of (1.1) we understand a function $Y:[a, \infty) \rightarrow \mathcal{L}(B)$ which is twice continuously differentiable in the uniform operator topology and satisfying (1.1) for all $t \in[a, \infty)$. We refer to the text of Hille [12] for a discussion of the concepts of differentiation and integration of functions from $[a, \infty)$ to a Banach algebra and to [15] for basic results concerning differential equations in Banach spaces. We shall be interested in comparing solutions of (1.1) with solutions of a second equation

$$
Y^{\prime \prime}+Q_{1}(t) Y=0
$$

with $Q_{1}:[a, \infty) \rightarrow \mathcal{L}(B)$ continuous. A solution $Y=Y(t)$ of (1.1) (or (1.2)) is said to be non-singular at a point $t_{0} \in[a, \infty)$ if it has a bounded inverse $Y^{-1}\left(t_{0}\right) \in \mathcal{L}(B)$. If $Y(t)$ is non-singular for all $t \in\left[t_{0},+\infty\right)$, some $t_{0} \geq a$, then $Y=Y(t)$ is said to be a non-oscillatory solution of (1.1) on $\left[t_{0},+\infty\right)$. Otherwise, $Y=Y(t)$ is said to be oscillatory on $[a, \infty)$. (Note that the inverse $Y^{-1}(t)$ of a non-oscillatory solution $Y(t)$ of (1.1) is continuously differentiable.)

Equations (1.1) and (1.2) have been the subject of numerous investigators ([4]-[11], [19], and the references therein) for the case that $B$ is a 
Hilbert space in which case $\mathcal{E}(B)$ is a $B^{*}$-algebra. Comparison and oscillation theorems have been obtained in many of the above references which are generalizations of the known theorems in the classical scalar case (e.g., the Sturm comparison theorem and the Hille-Wintner comparison theorem). In these results, the notion of comparison of operators in $\mathcal{L}(B)$ is used which is induced by the inner product; that is, if $T_{1}, T_{2} \in$ $\mathcal{L}(B)$ then $T_{1} \geq T_{2}$ means $\left\langle\left(T_{1}-T_{2}\right) x, x\right\rangle \geq 0$ for all $x$ in the Hilbert space $B$. In this paper we shall be interested in employing an alternate notion of positivity in $\mathcal{L}(B)$ which is induced by a vector lattice structure on $B$. This has the advantage in that the class of positive operators is not only a positive cone but is also closed under multiplication, a fact which is not true in the $B^{*}$-algebra case.

A further advantage of the Banach lattice context is that it enables one to obtain certain results for nonselfadjoint equations. Ahmad and Lazer ([1], [2]) have extended the Sturm comparison theorem to nonselfadjoint equations, and Ahmad and Travis [3] and Keener and Travis [14] have obtained oscillation criteria for such systems. The emphasis of the papers [1], [2] and [14] is on conjugate point and focal point properties.

Henceforth, $B$ will denote a Banach lattice. We recall that a Banach lattice $B$ is a Banach space with a vector lattice structure such that $|x| \leq|y|$ implies $\|x\| \leq\|y\|, x, y \in B$, where $|x| \equiv x_{\mathrm{v}}(-x)$ (see e.g. [17]). Let $B_{+}$be defined by $B_{+} \equiv\{x \in B: x \geq 0\}$. A subset $S \subset B$ is order bounded if there exists $z \in B_{+}$with $|x| \leq z$ for all $x \in S$. The Banach lattice $B$ is said to be order complete if for every non-empty majorized (with respect to the ordering) subset $A$ of $B, \sup A$ exists in $B$. Also, $B$ is said to have order continuous norm if each downward directed family $A$ of $B$ such that $\inf A=0$ converges in norm to 0 . A Banach lattice with order continuous norm is automatically order complete ([17], Theorem II.5.10, p. 89). Familiar examples of such Banach lattices are $l_{p}, \mathfrak{L}_{p}, 1 \leq p<\infty, c_{0}$, and any reflexive Banach lattice. Let $\mathcal{L}_{+}(B)$ denote the set of positive bounded linear operators on $B$. That is, $T \in \mathcal{L}_{+}(B)$ iff $T\left(B_{+}\right) \subset B_{+}$. An operator $T \in \mathcal{L}(B)$ is said to be regular if $T=T_{1}-T_{2}$ for some $T_{1}, T_{2} \in \mathcal{L}_{+}(B)$. $\mathcal{L}^{r}(B)$ denotes the vector space of regular operators on $B$. It can be shown (cf. [17], p. 229) that if $B$ is order complete, then $T$ is regular iff $T$ maps order bounded sets onto order bounded sets iff $|T| \equiv T_{\mathrm{v}}(-T)$ exists in the lattice structure induced on $\mathcal{L}(B)$. Furthermore, if $B$ is order complete, then $\operatorname{Lr}^{r}(B)$ is an order complete vector lattice ([17], p. 229). We refer to [16] and [17] for a more complete discussion of Banach lattices and their properties. 
The most familiar example of lattice structure is with $B=\mathbf{R}^{n}, B_{+}=$ $\left\{x \in \mathbf{R}^{n}: x_{l} \geq 0, i=1,2, \ldots, n\right\}, \varrho_{+}(B)=\left\{T: T\right.$ is an $n \times n$ matrix $\left(t_{l j}\right)$ with $\left.t_{i j} \geq 0 ; i, j=1,2, \ldots, n\right\}$. Another example is $B=l_{2}, B_{+}=\left\{x \in l^{2}\right.$ : $\left.x_{i} \geq 0, i=1,2, \ldots,\right\}$, where an important subset of $\mathcal{L}_{+}(B)$ is the set of positive Hilbert-Schmidt operators. These are compact operators $T$ for which $T e_{t} \in B_{+}, i=1,2, \ldots$, and $\sum_{l=1}^{\infty}\left\|T e_{i}\right\|^{2}<\infty$, where $\left\{e_{i}\right\}_{l=1}^{\infty}$ is the usual basis for $l^{2}$.

2. In this section it is our object to present in a more general setting some extensions of the classical Hille-Wintner comparison theorem for second-order scalar ordinary differential equations. The Hille-Wintner Theorem ([13], [20]) asserts that if $p(x)=\int_{x}^{\infty} q(t) d t, p_{1}(x)=\int_{x}^{\infty} q_{1}(t) d t$ exist (real-valued) and satisfy $\left|p_{1}(x)\right| \leq p(x)$ for all $x \in[a, \infty)$ then the existence of a non-oscillatory solution of

$$
y^{\prime \prime}(x)+q(x) y=0
$$

on $[a,+\infty)$ implies the existence of a non-oscillatory solution of

$$
y^{\prime \prime}(x)+q_{1}(x) y=0
$$

on $[a,+\infty)$. We shall consider the equations

$$
Y^{\prime \prime}+Q(t) Y=0
$$

and

$$
Y^{\prime \prime}+Q_{1}(t) Y=0
$$

and prove several generalizations of this result in the Banach lattice case. Etgen and Lewis [6] have extended the Hille-Wintner theorem in the $B^{*}$-algebra situation.

We begin with

THEOREM 2.1. Let B be a Banach lattice with order continuous norm. Suppose the limits

$$
P(t)=\lim _{T \rightarrow \infty} \int_{t}^{T} Q(s) d s, \quad P_{1}(t)=\lim _{T \rightarrow \infty} \int_{t}^{T} Q_{1}(s) d s
$$

exist (in the uniform operator topology of $\mathcal{L}(B)$ ) and

$$
P(t), P_{1}(t), P(t)-P_{1}(t) \in \mathcal{L}_{+}(B), \quad t \in[a,+\infty) .
$$

If there exists a non-oscillatory solution $Y(t)$ of (1.1) such that

$$
Z(t) \equiv Y^{\prime}(t) Y^{-1}(t) \in \mathcal{L}_{+}(B) \text { for all } t \in[a,+\infty),
$$

then (1.2) has a non-oscillatory solution on $[a,+\infty)$. 
Proof. Throughout, inequality signs will refer to the appropriate positive cone.

The Riccati transformation $Z(t)=Y^{\prime}(t) Y^{-1}(t)$ in (1.1) yields the equation

$$
Z(t)=Z(T)+\int_{t}^{T} Q(s) d s+\int_{t}^{T} Z^{2}(s) d s, \quad a \leq t<T<\infty .
$$

We assert that (2.3) implies that the set $\left\{\int_{t}^{T} Z^{2}(s) d s: a \leq T<\infty\right\}$ is an upward directed set in $\mathcal{L}^{r}(B)$ and is order bounded above by $Z(t)$. To see this, note first of all that the monotonicity of $\left\{\int_{t}^{T} Z^{2}(s) d s: a \leq T<\infty\right\}$ follows from the hypothesis that $Z(s)$ (and therefore $\left.Z^{2}(s)\right)$ is $\geq 0$ for all $s \geq a$. Denote $\int_{t}^{T} Z^{2}(s) d s$ by $\phi(t ; T)$. Then we can write $(2.3)$ as

$$
\phi(t ; T)=Z(t)-Z(T)-P(t)+P(T) .
$$

Then if $a \leq T \leq \tau$ we have, by (2.4) $\phi(t ; T) \leq \phi(t ; \tau) \leq Z(t)+P(\tau)$, so

$$
Z(t)+P(\tau)-\phi(t ; T) \geq 0, \quad a \leq T \leq \tau .
$$

For each $\tau \geq T$ the left-hand side of (2.5) is in $\mathcal{L}^{r}(B)$, which is a normed vector lattice and therefore has norm-closed positive cone $\mathcal{L}_{+}(B)$. Letting $\tau \rightarrow \infty$ in (2.5) we obtain $Z(t)-\phi(t ; T) \geq 0$, which completes the proof of our assertion. Since $\mathcal{L}^{r}(B)$ is order complete, it follows that $\phi(t) \equiv$ $\sup _{T} \phi(t ; T)$ exists. Let $x \in B_{+}$. Then (2.3) implies

$$
Z(T) x=Z(t) x-\left(\int_{t}^{T} Q(s) d s\right) x-\phi(t ; T) x .
$$

Since $B$ has order continuous norm, $\phi(t ; T) x \rightarrow \phi(t) x$ in norm, so $\phi(t)$ is the strong operator limit of $\phi(t ; T)$ as $T \rightarrow \infty$. Thus the limit in norm as $T \rightarrow \infty$ exists for the right-hand side of (2.6). We conclude that the limit in norm of $Z(T) x$ as $T \rightarrow \infty$ exists (in $B_{+}$by norm-closedness of $\left.B_{+}\right)$, call it $C(x)$. Thus

$$
C(x)=Z(t) x-P(t) x-\phi(t) x .
$$

It follows that $C(x)$ in fact defines a positive linear operator on $B_{+}$and extends uniquely to a linear operator on $B$ (cf. [17], p. 58). Thus, (2.7) holds for all $x$ and we may write $C(x)=C x$. Thus,

$$
Z(t)=C+P(t)+\phi(t), \quad C \in \mathcal{L}_{+}(B), t \in[a, \infty) .
$$


We now define a sequence of operator-valued functions $\left\{W_{n}(t)\right\}$ as follows:

Let $W_{0}(t) \equiv C, t \geq a$, and inductively

$$
\begin{aligned}
W_{n+1}(t) \equiv C+P_{1}(t)+\operatorname{s-lim}_{T \rightarrow \infty} \int_{t}^{T} W_{n}^{2}(s) d s, & \\
& n=0,1,2, \ldots, t \geq a,
\end{aligned}
$$

where s-lim denotes the strong operator limit. To justify this, we make the inductive assumption

$$
\left\{\begin{array}{l}
0 \leq W_{n}(t) \leq Z(t) \text { and } W_{n}(t) \text { is continuous in the } \\
\text { uniform operator topology of } \varrho(B), t \geq a .
\end{array}\right.
$$

Clearly $(2.10)_{0}$ is true. Assume (2.10) ${ }_{n}$ is true. Then $0 \leq W_{n}(s) \leq Z(s)$ implies $0 \leq W_{n}^{2}(s) \leq Z^{2}(s)$. This is a consequence of the cone $\mathscr{L}_{+}(B)$ being closed under multiplication. It does not, in general, hold in the $B^{*}$-algebra case. Thus $\left\{\int_{t}^{T} W_{n}^{2}(s) d s\right\}$ is monotone increasing in $T$ and is order bounded by $Z(t)$ since $\int_{t}^{T} W_{n}^{2}(s) d s \leq \int_{t}^{T} Z_{n}^{2}(s) d s \leq Z(t)$, and hence $s-\lim _{T \rightarrow \infty} \int_{t}^{T} W_{n}^{2}(s) d s$ exists. Thus $W_{n+1}(t)$ is well defined by $(2.9)_{n}$. It follows from the hypothesis $0 \leq P_{1}(t) \leq P(t)$ that $0 \leq W_{n+1}(t) \leq Z(t)$. We also have

$$
W_{n+1}(t)-W_{n+1}(s)=P_{1}(t)-P_{1}(s)-\int_{s}^{t} W_{n}^{2}(\tau) d \tau,
$$

so it follows that $W_{n+1}(t)$ is continuous, hence $(2.10)_{n+1}$ is true and thus $(2.10)_{n}$ is verified for all $n \geq 0$.

Let $\psi_{n}(t ; T)=\int_{t}^{T} W_{n}^{2}(s) d s$ and denote $s-\lim _{T \rightarrow \infty} \psi_{n}(t ; T)$ by $\psi_{n}(t)$. Since for fixed $t,\left\{W_{n}(t)\right\}_{n=1}^{\infty}$ is a monotone increasing sequence in $\mathcal{L}_{+}(B)$, bounded above by $Z(t)$, it follows that $\mathrm{s}-\lim _{n \rightarrow \infty} W_{n}(t)$ exists. Let $W(t) \equiv$ $\mathrm{s}-\lim _{n \rightarrow \infty} W_{n}(t)$. By $(2.9)_{n}$ we have

$$
W(t)=C+P_{1}(t)+\psi(t),
$$

where $\psi(t)=s-\lim _{n \rightarrow \infty} \psi_{n}(t)$. Note that $W(t)$ is continuous since

$$
W(t)-W(s)=P_{1}(t)-P_{1}(s)-\underset{n \rightarrow \infty}{\operatorname{sim}} \int_{s}^{t} W_{n}^{2}(\tau) d \tau
$$

and $0 \leq W_{n}(\tau) \leq Z(\tau)$ implies

$$
0 \leq\left\|\int_{s}^{t} W_{n}^{2}(s) d s\right\| \leq \int_{s}^{t}\left\|Z^{2}(s)\right\| d s \rightarrow 0 \quad \text { as } t-s \rightarrow 0
$$


by continuity of $Z$. For each $\tau$ and each $x \in B_{+}, W_{n}^{2}(\tau) x \rightarrow W^{2}(\tau) x$ in norm and $W^{2}(\tau) x$ is continuous in $\tau$. Now it follows from Dini's Theorem [21] that this convergence is uniform on any compact subinterval $[s, t]$ of $[a,+\infty)$. Thus

$$
\lim _{n \rightarrow \infty}\left(\int_{s}^{t} W_{n}^{2}(\tau) d \tau\right) x=\left(\int_{s}^{t} W^{2}(\tau) d \tau\right) x, \quad x \in B_{+},
$$

so that

$$
\operatorname{s}_{n \rightarrow \infty} \int_{s}^{t} W_{n}^{2}(\tau) d \tau=\int_{s}^{t} W^{2}(\tau) d \tau .
$$

We may therefore write (2.13) as

$$
W(t)-W(s)=P_{1}(t)-P_{1}(s)-\int_{s}^{t} W^{2}(\tau) d \tau .
$$

Dividing (2.14) by $t-s(t \neq s)$ and taking limits in the uniform operator topology as $s \rightarrow t$, we have

$$
W^{\prime}(t)=-Q_{1}(t)-W^{2}(t) .
$$

Now define $\hat{Y}(t)$ to be the solution of

$$
\hat{Y}^{\prime}(t)=W(t) \hat{Y}(t), \quad \hat{Y}(a)=I
$$

( $I=$ the identity operator in $\mathcal{L}(B)$ ). Then $\hat{Y}$ is a non-oscillatory solution of (1.2) and this proves the Theorem.

We note that Theorem 2.1 does allow for two operator-valued equations to be compared as distinct from the comparison theorems of [6] which compare an operator-valued equation with a scalar equation.

One would like to weaken hypothesis (i) in Theorem 2.1 to

(i) $\quad P_{1}(t) \in \mathcal{L}^{r}(B), \quad P(t), \quad P(t)-\left|P_{1}(t)\right| \in \mathcal{L}_{+}(B), \quad t \in[a,+\infty)$,

and to dispense with hypothesis (ii) (which holds automatically in the scalar case for a non-oscillatory solution $Y(t)$ of $(1.1)$ when $P(t) \geq 0)$.

As far as (ii) is concerned, we may make the following remarks:

Assume $P(t) \in \mathfrak{L}^{+}(B), t \in[a, \infty)$. Define $P_{0}(t)$ to be $P(t)$ and $P_{n}(t)$ by $P_{n}(t)=P(t)+\mathrm{s}-\lim _{T \rightarrow \infty} \int_{t}^{T} P_{n-1}^{2}(s) d s$. Then the existence of a nonoscillatory solution $Y(t)$ of (1.1) satisfying (ii) is equivalent to

$$
\begin{aligned}
& P_{n}(t) \text { is defined for all } t \in[a, \infty), n=0,1,2, \ldots, \text { and } \\
& s-\lim _{n \rightarrow \infty} P_{n}(t)=\hat{P}(t) \text { exists. }
\end{aligned}
$$

The equivalence of (ii) and (iii) is established by essentially the same arguments as were used in the proof of Theorem 2.1 and is an extension of 
a well-known result in the scalar case (see [18], Theorem 3.2, where the scalar result is given, though expressed in a slightly different form). One marked difference from the scalar case, however, is that there may be nonoscillatory solutions $Y(t)$ of (1.1) for which (ii) does not hold, as the following example illustrates. Let $B=\mathbf{R}^{2}$ and take

$$
Q(t)=\frac{1}{288 t^{2}}\left(\begin{array}{cc}
24 & 5 \\
5 & 48
\end{array}\right)
$$

Then

$$
Y(t)=\frac{1}{5}\left(\begin{array}{cc}
t^{\mu} & -25 t^{\nu} \\
5 t^{\mu} & 5 t^{\nu}
\end{array}\right)
$$

is a solution of (1.1), where

$$
\mu=\frac{1}{2}\left(1-\sqrt{\frac{23}{72}}\right), \quad \nu=\frac{1}{2}\left(1+\sqrt{\frac{49}{72}}\right) .
$$

It may be verified that

$$
Z(t)=Y^{\prime}(t) Y^{-1}(t)=\frac{1}{26 t}\left(\begin{array}{cc}
\mu+5 \nu & -5(\nu-\mu) \\
-5(\nu-\mu) & \nu+5 \mu
\end{array}\right),
$$

so $Z(t) \notin \mathcal{L}_{+}(B)$. In this case however, (1.1) does have a (different) nonoscillatory solution $Y(t)$ for which (ii) holds, namely

$$
Y(t)=\frac{1}{5}\left(\begin{array}{cc}
t^{\alpha} & -25 t^{\beta} \\
5 t^{\alpha} & 5 t^{\beta}
\end{array}\right), \text { where } \alpha=\frac{1}{2}\left(1+\sqrt{\frac{23}{72}}\right), \beta=\frac{1}{2}\left(1-\sqrt{\frac{49}{72}}\right) \text {. }
$$

We do not know whether $P(t) \in \mathcal{L}_{+}(B), t \in[a, \infty)$, and the existence of a nonoscillatory solution of (1.1) together do imply that (ii) holds for some nonoscillatory solution $Y(t)$ (and therefore that (iii) holds).

In the case that $B$ is finite dimensional, we can replace (i) by (i)'.

TheOREM 2.2. Let $B=R^{n}$ and suppose

$$
P(t)=\lim _{T \rightarrow \infty} \int_{t}^{T} Q(s) d s, \quad P_{1}(t)=\lim _{T \rightarrow \infty} \int_{t}^{T} Q_{1}(s) d s
$$

exist such that (i)' holds. If there exists a non-oscillatory solution $Y(t)$ of (1.1) such that (ii) holds, then (1.2) has a non-oscillatory solution on $[a, \infty)$.

Proof. As in the proof of Theorem 2.1, we put $Z(t)=Y^{\prime}(t) Y^{-1}(t)$ in (1.1) and obtain

$$
Z(t)=Z(T)+\int_{t}^{T} Q(s) d s+\int_{t}^{T} Z^{2}(s) d s, \quad a \leq t \leq T<\infty,
$$


which leads to

$$
Z(t)=C+P(t)+\phi(t), \quad t \in[a, \infty),
$$

where

$$
C \in \mathcal{L}_{+}(B), \quad \phi(t)=\int_{t}^{\infty} Z^{2}(s) d s \in \mathcal{L}_{+}(B), \quad t \in[a, \infty) .
$$

Let be the set of continuous functions $W:[a, \infty) \rightarrow \mathscr{L}\left(R^{n}\right)$ such that $|W(t)| \leq Z(t)$ for all $t \in[a, \infty)$.

Then $\mathscr{W}$ is a closed, convex subset of $\mathfrak{K}$, the Fréchet space of continuous operator-valued functions on $[a, \infty)$ with the compact-open topology.

Now if $W \in \mathscr{W}$, then for each $t \in[a, \infty), W(t)$ may be represented by an $n \times n$ matrix, say $W(t)=\left[(W(t))_{i j}\right]$. Similarly we may represent $Z(t)$ by the $n \times n$ matrix $\left[(Z(t))_{i j}\right]$, and we have

$$
\left|(W(t))_{i j}\right| \leq(Z(t))_{i j}, \quad i, j=1, \ldots, n .
$$

It follows that $\left|W^{2}(t)\right| \leq Z^{2}(t)$. Furthermore, we have $\left\|W^{2}(t)\right\| \leq\left\|Z^{2}(t)\right\|$, where $\|\cdot\|$ denotes the uniform operator norm on $\mathcal{L}(B)$. Since $Z^{2}(t)$ is integrable on $[a, \infty)$, it follows that $W^{2}(t)$ is integrable on $[a, \infty)$, and

$$
\left|\int_{t}^{\infty} W^{2}(s) d s\right| \leq \int_{t}^{\infty} Z^{2}(s) d s
$$

It follows from (2.9), (2.17) and (i)' that the map $F$ on $थ$ defined by

$$
(F W)(t)=C+P_{1}(t)+\int_{t}^{\infty} W^{2}(s) d s
$$

is a map from $\mho$ into itself.

Let $\left\{W_{n}\right\}_{n=1}^{\infty}, W \in \mathcal{W}$ with $\lim _{n \rightarrow \infty} W_{n}=W$. For any $T \geq t>a$, we have

$$
\begin{gathered}
\left\|\left(F W_{n}\right)(t)-(F W)(t)\right\|=\left\|\int_{t}^{\infty} W_{n}^{2}(s) d s-\int_{t}^{\infty} W^{2}(s) d s\right\| \\
\leq \int_{t}^{T}\left\|W_{n}^{2}(s)-W^{2}(s)\right\| d s+\left\|\int_{T}^{\infty} W_{n}^{2}(s) d s\right\| \\
\quad+\left\|\int_{T}^{\infty} W^{2}(s) d s\right\| .
\end{gathered}
$$

Since

$$
\left\|\int_{T}^{\infty} W_{n}^{2}(s) d s\right\| \leq\left\|\int_{T}^{\infty} Z^{2}(s) d s\right\|
$$


and

$$
\left\|\int_{T}^{\infty} W^{2}(s) d s\right\| \leq\left\|\int_{T}^{\infty} Z^{2}(s) d s\right\|
$$

we may, for any given $\varepsilon>0$, choose $T=T(\varepsilon)$ so that

$$
\left\|\int_{T}^{\infty} W_{n}^{2}(s) d s\right\|<\frac{\varepsilon}{3}, \quad n=1,2, \ldots,
$$

and

$$
\left\|\int_{T}^{\infty} W^{2}(s) d s\right\|<\frac{\varepsilon}{3} .
$$

By the uniform convergence of $W_{n}$ to $W$ on $[a, T]$ we have

$$
\int_{t}^{T}\left\|W_{n}^{2}(s)-W^{2}(s)\right\| d s<\frac{\varepsilon}{3} \quad \text { for all } n \geq N(\varepsilon),
$$

say, and for all $t \in[a, T]$. Thus (2.19) gives

$$
\left\|\left(F W_{n}\right)(t)-(F W)(t)\right\|<\varepsilon \text { for all } n \geq N(\varepsilon), \quad t \in[a, \infty) .
$$

It follows that $\lim _{n \rightarrow \infty} F W_{n}=F W$, that is, $F:$ $\circlearrowleft \rightarrow$ is continuous.

Now for $s, t \geq a$, we have

$$
\|F W(t)-F W(s)\| \leq \int_{s}^{t}\left\|Q_{1}(\tau)\right\| d \tau+\int_{s}^{t}\left\|Z^{2}(\tau)\right\| d \tau .
$$

It follows that $F(\mho)$ consists of equicontinuous, uniformly bounded operator-valued functions. Thus $F(\mathcal{Q})$ is precompact.

By Tychonov's Theorem ([10], p. 405), $F$ has a fixed point in 25 , that is, there exists $W(t)$ such that

$$
W(t)=C+P_{1}(t)+\int_{t}^{\infty} W^{2}(s) d s .
$$

It follows that (1.2) has a non-oscillatory solution.

Our next result is of a somewhat different nature. Here we compare the operator equation (1.2) in $\mathcal{E}\left(R^{n}\right)$ with an appropriate scalar equation

$$
y^{\prime \prime}(t)+q(t) y(t)=0 \text {. }
$$

TheOrem 2.3. Let $Q_{1}:[a, \infty) \rightarrow \mathcal{L}\left(R^{n}\right)$ be continuous and let $\left[\left(Q_{1}(t)\right)_{l j}\right]$ be the usual matrix representation of $Q_{1}(t)$. Assume

$$
\left(P_{1}(t)\right)_{i j}=\int_{t}^{\infty}\left(Q_{1}(s)\right)_{i j} d s
$$


exists for all $i, j$. Let $q:[a, \infty) \rightarrow R$ be continuous and suppose $p(t)=$ $\int_{t}^{\infty} q(s) d s$ exists, with

$$
p(\sqrt{n} t) \geq \sqrt{n} \max _{i, j}\left(P_{1}(t)\right)_{i j}, \quad t \in[a, \infty) .
$$

If (2.21) has a non-oscillatory solution on $[a, \infty)$, then so does (1.2).

Proof. Let $y(t)$ be a non-oscillatory solution of (2.21) and put $u(t)=$ $y^{\prime}(t) y^{-1}(t)$ to obtain the Riccati equation

$$
u^{\prime}(t)=-q(t)-u^{2}(t) \text {. }
$$

Put $v(t)=u(\sqrt{n} t) / \sqrt{n}$ to obtain

$$
v^{\prime}(t)=-q(\sqrt{n} t)-n v^{2}(t) .
$$

Integrate (2.22) to obtain

$$
v(t)=v(T)+\int_{t}^{T} q(\sqrt{n} s) d s+n \int_{t}^{T} v^{2}(s) d s .
$$

Letting $T \rightarrow \infty$ in (2.23), it follows that $\lim _{T \rightarrow \infty} v(T)=b$, where $-\infty \leq b$ $<\infty$. In fact, it is easy to show that $0 \leq b<\infty$, and we have

$$
v(t)=b+\frac{1}{\sqrt{n}} p(\sqrt{n} t)+n \int_{t}^{\infty} v^{2}(s) d s .
$$

Let $\mho_{0}$ be the set of continuous functions $W(t)=\left[(W(t))_{i j}\right]$ from $[a, \infty)$ to $\mathcal{L}\left(R^{n}\right)$ such that $\left|(W(t))_{i j}\right| \leq v(t), t \in[a, \infty)$, for each $i, j$.

Define the map $F_{0}: \mathscr{Q}_{0}$ to $\mathscr{W}_{0}$ by

$$
\begin{array}{r}
\left(\left(F_{0} W\right)(t)\right)_{i j}=\left(P_{1}(t)\right)_{i j}+\int_{t}^{\infty}\left(\sum_{k=1}^{n}(W(s))_{i k}(W(s))_{k j}\right) d s, \\
t \in[a, \infty), i, j=1, \ldots, n .
\end{array}
$$

It follows from hypothesis (iii) and (2.24) that $F_{0}$ is well defined. $\mho_{0}$ is a closed, convex subset of $\Re$, the Fréchet space of continuous operatorvalued functions on $[a, \infty)$ with the compact-open topology. Arguments similar to those used in the proof of Theorem 2.2 show that $F_{0}$ is continuous on $\mho_{0}$ and that $F_{0}\left(\mho_{0}\right)$ is a precompact subset of $\mho_{0}$. Hence Tychonov's theorem yields the existence of a fixed point for $F_{0}$, that is, there exists $W(t)=\left[(W(t))_{i j}\right]$ such that

$$
\begin{array}{r}
(W(t))_{i j}=\left(P_{1}(t)\right)_{\imath j}+\int_{t}^{\infty}\left(\sum_{k=1}^{n}(W(s))_{\imath k}(W(s))_{k j}\right) d s \\
t \in[a, \infty) ; i, j=1, \ldots, n .
\end{array}
$$


By examining the matrix Riccati equation for (1.2), it is apparent that (2.26) enables us to define a non-oscillatory solution of (1.2).

3. We now present two examples which illustrate the results obtained.

EXAMPLE 3.1. Let $B$ be a Banach lattice with order continuous norm and let $B^{\prime}$ be its dual space. ( $B^{\prime}$ is also a Banach lattice, under the dual norm, which is order complete.) Let $c \in B_{+}$and $\phi \in B_{+}^{\prime}$. Let $q(t)$ be a real-valued positive, twice continuously differentiable function on $[a,+\infty)$ such that $q^{\prime}(t)>0$ and $q^{\prime \prime}(t)<0$ for all $t \in[a,+\infty)$. Define $Q:[a, \infty)$ $\rightarrow \mathfrak{L}(B)$ by

$$
Q(t) x=\frac{-q^{\prime \prime}(t) \phi(x) c}{1+q(t) \phi(c)}, \quad x \in B
$$

Then

$$
Q(t) \in \varrho_{+}(B) \quad \text { for all } t \in[a, \infty) \quad\left(\|Q(t)\|=\frac{\left|q^{\prime \prime}(t)\right| \cdot\|\phi\| \cdot\|c\|}{1+q(t)\|\phi(c)\|}\right) .
$$

Note that

$$
\left\|\int_{t}^{\infty} Q(s) d s\right\|=\left(\int_{t}^{\infty} \frac{-q^{\prime \prime}(s) d s}{1+q(s)(c)}\right)\|\phi\| \cdot\|c\| \leq q^{\prime}(t)\|\phi\| \cdot\|c\| .
$$

It may be verified that $Y^{\prime \prime}+Q(t) Y=0$ has a solution $Y(t)$ defined by

$$
Y(t) x=x+q(t) \phi(x) c, \quad x \in B .
$$

Since

$$
Y^{-1}(t) x=x-\frac{q(t) \phi(x) c}{1+q(t) \phi(c)}, \quad x \in B
$$

it follows that $Y(t)$ is a non-oscillatory solution and, furthermore, the Riccati variable $Z(t)=Y^{\prime}(t) Y^{-1}(t)$ is given by

$$
Z(t) x=\frac{q^{\prime}(t) \phi(x) c}{1+q(t) \phi(c)}, \quad x \in B
$$

so $Z(t) \in \mathcal{L}_{+}(B)$ on $[a, \infty)$. Therefore, if $c_{1} \in B_{+}, c_{1} \leq c, \phi_{1} \in B_{+}^{\prime}$, $\phi_{1} \leq \phi$, and if $q_{1}(t)$ is a real-valued function such that

$$
0 \leq \int_{t}^{\infty} \frac{-q_{1}^{\prime \prime}(s)}{1+q_{1}(s) \phi_{1}\left(c_{1}\right)} d s \leq \int_{t}^{\infty} \frac{-q^{\prime \prime}(s)}{1+q(s) \phi(c)} d s, \quad t \in[a,+\infty),
$$


then the equation $Y^{\prime \prime}+Q_{1}(t) Y=0$ has a non-oscillatory solution by Theorem 2.1, where $Q_{1}(t)$ is given by

$$
Q_{1}(t) x=\frac{-q_{1}^{\prime \prime}(t) \phi_{1}(x) c_{1}}{1+q_{1}(t) \phi_{1}(c)}, \quad x \in B
$$

Note that $Q_{1}(t) \notin \mathcal{L}_{+}(B)$ in general.

EXAMPLE 3.2. As an application of Theorem 2.2, consider the case $n=2$. Let

$$
Q(t)=t^{-2}\left(\begin{array}{ll}
a & b \\
c & d
\end{array}\right) \quad \text { where } a, b, c, d \geq 0, \quad t \in[1, \infty) .
$$

Invoking condition (iii) (see the remarks following Theorem 2.1), there is a nonoscillatory solution $Y(t)$ of (1.1) satisfying (ii) iff the sequence $P_{n}(t)$ converges as $n \rightarrow \infty$, where

$$
\begin{aligned}
& P_{0}(t)=P(t)=t^{-1}\left(\begin{array}{ll}
a & b \\
c & d
\end{array}\right), \\
& P_{n}(t)=P(t)+\int_{t}^{\infty} P_{n-1}^{2}(s) d s, \quad n=1,2, \ldots
\end{aligned}
$$

If

$$
P_{n}(t)=t^{-1}\left(\begin{array}{ll}
a_{n} & b_{n} \\
c_{n} & d_{n}
\end{array}\right)
$$

we obtain the relations

$$
\begin{aligned}
& a_{n+1}=a+a_{n}^{2}+b_{n} c_{n}, \\
& b_{n+1}=b+b_{n}\left(a_{n}+d_{n}\right), \\
& c_{n+1}=c+c_{n}\left(a_{n}+d_{n}\right), \\
& d_{n+1}=d+d_{n}^{2}+b_{n} c_{n}, \quad n=0,1, \ldots .
\end{aligned}
$$

Since $a, b, c, d \geq 0$, the sequences $a_{n}, b_{n}, c_{n}, d_{n}$ are increasing with $n$ and have finite limits $\alpha, \beta, \gamma, \delta$ iff the system of equations

$$
\begin{aligned}
& \alpha=a+\alpha^{2}+\beta \gamma, \\
& \beta=b+\beta(\alpha+\delta), \\
& \gamma=c+\gamma(\alpha+\delta), \\
& \delta=d+\delta^{2}+\beta \gamma
\end{aligned}
$$

has a solution. 
After some manipulation of these equations, we find that solvability of (2.28) is equivalent to the existence of a positive solution $\theta$ of the equation

$$
\theta^{2}\left\{1-2(d+a)-\theta^{2}\right\}=(d-a)^{2}+4 b c .
$$

$\alpha, \beta, \gamma, \delta$ are then given by

$$
\alpha=\frac{1}{2}\left(1-\theta-\frac{d-a}{\theta}\right), \quad \beta=\frac{b}{\theta}, \quad \gamma=\frac{c}{\theta}, \quad \delta=\frac{1}{2}\left(1-\theta+\frac{d-a}{\theta}\right) .
$$

This, in turn, is easily seen to be equivalent to the condition

$$
\left\{\begin{array}{l}
a<\frac{1}{4}, \quad \text { if } d=a \text { and } b c=0 \\
d+a+\sqrt{(d-a)^{2}+4 b c} \leq \frac{1}{2}, \quad \text { if } d \neq a \text { or } b c \neq 0 .
\end{array}\right.
$$

Let $a, b, c, d$ be any nonnegative constants satisfying (2.30) with equality. Now let $Q_{1}(t)=\left[q_{i j}(t)\right]$ be chosen so that

$$
\varlimsup_{t \rightarrow \infty} t \int_{t}^{\infty} q_{11}(s) d s<a, \quad \varlimsup_{t \rightarrow \infty} t \int_{t}^{\infty} q_{12}(s) d s<b,
$$

$$
\begin{aligned}
& \varlimsup_{t \rightarrow \infty} t \int_{t}^{\infty} q_{21}(s) d s<c, \\
& \varlimsup_{t \rightarrow \infty} t \int_{t}^{\infty} q_{22}(s) d s<d .
\end{aligned}
$$

Then by Theorem 2.2, (1.2) has a non-oscillatory solution.

This example may be thoughout of as an extension to the $2 \times 2$ matrix case of Hille's non-oscillation criterion [13]

$$
\varlimsup_{t \rightarrow \infty} t q_{1}(t)<1 / 4
$$

for the scalar equation

$$
y^{\prime \prime}+q_{1}(t) y=0 .
$$

In case $b=c=d=0$, we have $a=1 / 4$ and (2.31) reduces to (2.32). Other examples can be readily found utilizing condition (iii).

\section{REFERENCES}

[1] S. Ahmad and A. C. Lazer, An n-dimensional extension of the Sturm separation and comparison theory to a class of nonselfadjoint systems, SIAM J. Math. Anal., 9 (1978), 1137-1150.

[2] On an extension of Sturm's comparison theorem to a class of nonselfadjoint second order systems, Nonlinear Analysis, 4 (1980), 497-501. 
[3] S. Ahmad and C. C. Travis, Oscillation criteria for second order differential systems, Proc. Amer. Math. Soc., 71 (1978), 247-252.

[4] W. Allegretto and L. Erbe, Oscillation criteria for matrix differential inequalities, Canad. Math. Bull., 16 (1973), 5-10.

[5] G. J. Etgen and R. T. Lewis, Positive functionals and oscillation criteria for second-order differential systems, Proc. Edinburgh Math. Soc., 22 (1979), 277-290. A Hille-Wintner comparison theorem for second order differential systems, Czech. Math. J., 30 (1980), 98-107.

[7] The oscillation of ordinary differential equations in a $B^{*}$-algebra, (preprint).

[8] G. J. Egen and J. F. Pawlowski, Oscillation criteria for second-order self-adjoint differential systems, Pacific J. Math., 66 (1976), 99-110.

[9] _ A comparison theorem and oscillation criteria for second-order differential systems, Pacific J. Math., 72 (1977), 59-69.

[10] P. Hartman, Ordinary Differential Equations, Wiley, New York, 1964.

[11] T. L. Hayden and H. C. Howard, Oscillation of differential equations in Banach spaces, Annali di Mathematica Pura ed Applicata, 85 (1970), 383-394.

[12] E. Hille, Lectures on Ordinary Differential Equations, Addison-Wesley, Reading, Mass., 1969.

[13] , Non-oscillation theorems, Trans. Amer. Math. Soc., 64 (1948), 234-252.

[14] M. S. Keener and C. C. Travis, Sturmian theory for a class of nonselfadjoint differential systems, Annali di Mathematica Pura ed Applicata, 123 (1980), 247-266.

[15] R. H. Martin, Nonlinear Operators and Differential Equations in Banach Spaces, Wiley, New York, 1976.

[16] A. P. Robertson and W. Robertson, Topological Vector Spaces, 2nd. ed., Cambridge University Press, 1973.

[17] H. H. Schaefer, Banach Lattices and Positive Operators, Springer, Berlin-HeidelbergNew York, 1974.

[18] D. Willett, $A$ necessary and sufficient condition for the oscillation of some linear second order differential equations, Rocky Mountain J. Math., 1 (1971), 357-365.

[19] C. M. Williams, Oscillation phenomena for linear differential equations in a $B^{*}$-algebra, Ph.D. dissertation. University of Oklahoma, 1971.

[20] A. Wintner, On the comparison theorem of Kneser-Hille, Math. Scand., 56 (1957), $255-260$.

[21] A. C. Zaanen, Linear Analysis, P. Noordhoff, Groningen, and Interscience Pub., New York, 1953.

Received January 13, 1981 and in revised form June 11, 1982. Research supported by NSERC Grants A-8130 and A-7673.

UNIVERSITY OF ALBERTA

Edmonton, Alberta, Canada T6G 2H1 


\section{PACIFIC JOURNAL OF MATHEMATICS \\ EDITORS}

DONALD BABBITT (Managing Editor)

University of California.

Los Angeles, CA 90024

Hugo Rossi

University of Utah

Salt Lake City, UT 84112

C. C. Moore and Arthur Ogus

University of California

Berkeley, CA 94720
J. DugundjI

Department of Mathematics

University of Southern California

Los Angeles, CA 90089-1113

R. FinN and H. Samelson

Stanford University

Stanford, CA 94305

\section{ASSOCIATE EDITORS}
R. ARENS
E. F. BECKENBACH
B. H. NeumanN
F. WOLF
K. YoshidA (1906-1982)

\section{SUPPORTING INSTITUTIONS}

UNIVERSITY OF ARIZONA

UNIVERSITY OF BRITISH COLUMBIA

CALIFORNIA INSTITUTE OF TECHNOLOGY

UNIVERSITY OF CALIFORNIA

MONTANA STATE UNIVERSITY

UNIVERSITY OF NEVADA. RENO

NEW MEXICO STATE UNIVERSITY

OREGON STATE UNIVERSITY
UNIVERSITY OF OREGON

UNIVERSITY OF SOUTHERN CALIFORNIA

STANFORD UNIVERSITY

UNIVERSITY OF HAWAII

UNIVERSITY OF TOKYO

UNIVERSITY OF UTAH

WASHINGTON STATE UNIVERSITY

UNIVERSITY OF WASHINGTON 


\section{Pacific Journal of Mathematics}

Vol. 112, No. $1 \quad$ January, 1984

Richard Blaine Barrar and Henry Loeb, Characterizing the divided difference weights for extended complete Tchebycheff systems $\ldots \ldots \ldots \ldots 1$

Harold Bennett and David John Lutzer, Generalized ordered spaces with

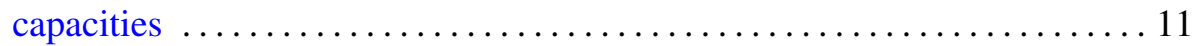

Geoffrey J. Butler and Lynn Harry Erbe, Comparison theorems for second-order operator-valued linear differential equations

Bohumil Cenkl and Richard D. Porter, de Rham theorem with cubical

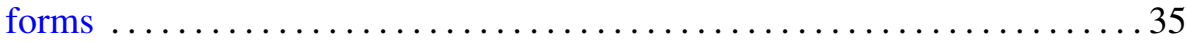

Zvonko Cerin, Characterizing global properties in inverse limits . ....... 49

Thomas Eugene Duchamp and Morris Kalka, Holomorphic foliations and deformations of the Hopf foliation .........................69 69

John Paul Hempel, Homology of coverings $\ldots \ldots \ldots \ldots \ldots \ldots \ldots \ldots \ldots$

Gerald Norman Hile and R. Z. Yeh, Inequalities for eigenvalues of the

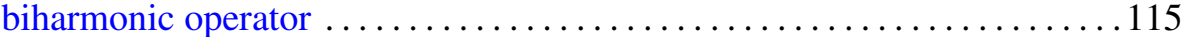

Kenneth Irwin Joy, A description of the topology on the dual space of a

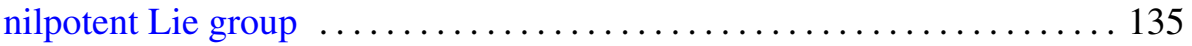

Alex Kumjian, On localizations and simple $C^{*}$-algebras $\ldots \ldots \ldots \ldots \ldots 141$

Bernardus de Pagter, The space of extended orthomorphisms in a Riesz

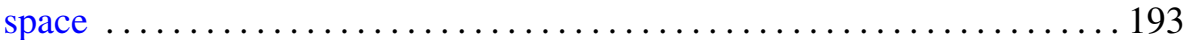

Stephen C. Persek, Iterated averaging for periodic systems with hidden

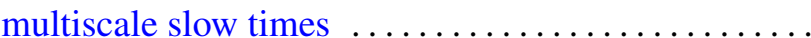

David Francis Rearick, Divisibility of arithmetic functions . . . . . . . . . 237

Masaaki Suzuki, The intrinsic metrics on the circular domains in $\mathbf{C}^{n}$ 